\title{
1 Pharmacological Profile of Vascular Activity of Human Stem Villous
}

2

3

4

5

6

7

8

9

\section{Arteries}

Katrin N Sander, Tayyba Y Ali, Averil Y Warren, Daniel P Hay, Fiona Broughton Pipkin, David A Barrett, Raheela N Khan.

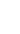

Affiliations:

Division of Medical Science and Graduate Entry Medicine, School of Medicine, University of Nottingham, The Royal Derby Hospital, Uttoxeter Road, Derby, DE22 3DT, UK (K.N.S., T.Y.A., A.Y.W., D.P.H., R.N.K.); Obstetrics \& Gynaecology, Faculty of Medicine, University of Nottingham, Queen's Medical Centre, Nottingham, NG7 2UH, UK (F.B.P.); Advanced Materials and Healthcare Technologies Division, Centre for Analytical Bioscience, School of Pharmacy, University of Nottingham, University Park, Nottingham, NG7 2RD, UK (K.N.S., D.A.B).

Correspondence to Raheela N. Khan, Division of Medical Science and Graduate Entry Medicine, School of Medicine, University of Nottingham, Royal Derby Hospital, Uttoxeter Road, Derby, DE22 3DT, UK. E-mail: raheela.khan@nottingham.ac.uk.Phone: +44 1332724664

Keywords:

Pregnancy, human

Placenta

Vascular function

Wire myography

Stem villous arteries

Placental vessels 
25

\section{Abstract}

Introduction

The function of the placental vasculature differs considerably from other systemic vascular beds of the human body. A detailed understanding of the normal placental vascular physiology is the foundation to understand perturbed conditions potentially leading to placental dysfunction.

\section{Methods}

Behaviour of human stem villous arteries isolated from placentae at term pregnancy was assessed using wire myography. Effects of a selection of known vasoconstrictors and vasodilators of the systemic vasculature were assessed. The morphology of stem villous arteries was examined using IHC and TEM.

\section{Results}

Contractile effects in stem villous arteries were caused by U46619, 5-HT, angiotensin II and endothelin-1 ( $p \leq 0.05)$, whereas noradrenaline and AVP failed to result in a contraction. Dilating effects were seen for histamine, riluzole, nifedipine, papaverine, SNP and SQ29548 ( $p \leq 0.05)$ but not for acetylcholine, bradykinin and substance $P$.

\section{Discussion}

Stem villous arteries behave differently to vessels of the systemic vasculature and results indicate that the placenta is cut off from the systemic maternal vascular regulation. Particularly, endothelium-dependent processes were attenuated in the placental vasculature, creating a need to determine the role of the endothelium in the placenta in future studies. 
46

47

\section{Introduction}

Placental vessels are of low resistance and their control is mainly driven by local humoral factors [1].

Due to the lack autonomic innervation, many vasoactive substances of the systemic vasculature exhibit no effects in placental vessels [2]. This 'failsafe' function of placental vessels ensures sufficient blood flow to the fetus at any time, independent from factors affecting the maternal organism.

There are two types of vessels in the placenta that exhibit characteristics of resistance arteries with normalised internal diameters of 100-400 $\mu \mathrm{m}$ [3] and muscular walls [4]: chorionic plate arteries and stem villous arteries. Stem villous arteries are situated at the site of nutrient transfer and are also present in much higher numbers than chorionic plate arteries. Stem villous arteries are therefore thought to be the most significant structure for the regulation of the placental circulation [5]. Although chorionic plate arteries may be less important for direct regulation of the fetoplacental flow, they might affect the downstream vasculature by release of mediators [6].

Several publications report the effect of various pharmacological compounds on placental vessels [712]. However, most of the recent literature on placental vessels focusses on chorionic plate arteries whereas knowledge about stem villous arteries dates back to research conducted in the 1990s. This early work was mostly performed using perfusion of whole placentae or isolated vessels, often under nonphysiological conditions using high oxygen pressures and high resting tensions, potentially leading to distorted findings. Therefore, the present study undertook to test the effect of a selection of known vasoconstrictors and vasodilators on stem villous arteries under physiological experimental conditions. 
67

68

69

\section{Methods}

\section{Chemicals and Solutions}

Two types of buffers were used for wire myography experiments, physiological salt solution (PSS) and high potassium physiological salt solution (KPSS). The composition of PSS (in mM) is sodium chloride 119 , potassium chloride 4.7 , magnesium sulfate heptahydrate 1.17 , sodium bicarabonate 25 , potassium dihydrogen orthophosphate 1.18 , EDTA 0.027 , D-(+)-glucose 5.5 , calcium chloride dehydrate 2.5. For KPSS, sodium chloride was replaced with $123.7 \mathrm{mM}$ potassium chloride. Both were prepared according to protocols developed by Mulvany [3]. (R)-(-)-Phenylephrine hydrochloride (P6126), [ $\left.\mathrm{Arg}^{8}\right]$-Vasopressin acetate salt (V9879), acetylcholine chloride (A6625), angiotensin II human (A9525), bradykinin acetate salt (B3259), histamine (H7125), indomethacin (17378), L-norepinephrine hydrochloride (74480), nifedipine (N7634), N $\Omega$-nitro-L-arginine methyl ester hydrochloride (L-NAME) (N5751), papaverine hydrochloride (P3510), riluzole (R116), sodium nitroprusside dehydrate (S0501) and Substance P acetate salt hydrate (S6883) were bought from Sigma, UK. U46619 (1932) was bought from Tocris, UK. Endothelin-1 (human, porcine) (ab120471) was purchased from Abcam, UK. Serotonin (hydrochloride) (14332) was bought from Cayman, US. SQ29548 (BML-RA103) was purchased from Enzo, UK.

\section{Tissue collection}

Placentae were collected from healthy pregnant women after obtaining fully informed written consent. Ethics approval was granted by Derby Research Ethics Committee (REC Reference No. 09/H0401/90). Patient demographics for collected placentae are shown in Table 1. All subjects of the study delivered via caesarean section.

\section{Wire myography}

Stem villous arteries were dissected within one hour after collection and placed in physiological salt solution (PSS). In order to accurately identify arteries in stem villi for the purposes of myography, the umbilical artery from the point of cord insertion was followed to first excise a full cotyledon. The cotyledon was cleaned from excess blood using PSS and the artery was then followed directly while 
continuously removing surrounding villous tissue using blunt forceps and a fine pair of scissors, taking extreme care not to damage the vessel wall. From the third to fourth order of the branch, dissection needed to be continued under the dissecting microscope in order to distinguish the artery from the vein that usually runs in close proximity within a villus. The arteries were cleaned from surrounding tissue and cut into $2 \mathrm{~mm}$ segments. Vessel segments were mounted onto $40 \mu \mathrm{m}$ wires of a DMT 620 myograph system (Aarhus, Denmark) and normalised. For the present study, all vessels were normalised to a target pressure of $5.1 \mathrm{kPa}$ in order to simulate physiological placental conditions [10]. An internal circumference of $0.9 * \mid C_{5.1 \mathrm{kPa}}$ was used as optimal working diameter for stem villous arteries. Experiments were performed at $37^{\circ} \mathrm{C}$ in PSS gassed with $2 \%$ oxygen, $5 \%$ carbon dioxide in nitrogen (BOC special gas, British Oxygen Company, UK) to reproduce placental conditions at term [13].

Experiments were started with an initial contraction to $10^{-6} \mathrm{M}$ U46619 that served as a viability control. Effects of subsequently tested contractile or relaxant agents were expressed in $\%$ of this contraction. For the assessment of relaxant effects, test compounds were added in increasing concentrations directly following the initial U46619 contraction. U44619 was chosen as its vasoconstrictive effects have been shown to be consistent and reproducible in placental vessels [10]. For assessment of contractile effects, the initial U46619 dose was washed out and the vessels left to equilibrate to baseline tension before adding increasing concentrations of a test compound. Every experiment was completed with a final contraction by changing the buffer from PSS to KPSS to confirm viability.

\section{Immunohistochemistry (IHC)}

$1 \mathrm{~mm}$ long stem villous arteries post myography were placed immediately placed in Bouin's solution overnight at $4^{\circ} \mathrm{C}$. Following fixation, samples were mounted in Optimum Cutting Temperature (OCT) embedding medium (Tissue Tek) and rapidly frozen in liquid $\mathrm{N}_{2}$ cooled isopentane. The freshly frozen samples were then transferred to a cryostat maintained at $-18^{\circ} \mathrm{C}$ (Leica CM1900) and sectioned to give $5 \mu \mathrm{m}$ thick slices. The sections were then adhered to a gelatine-coated slide $(76 \times 26 \times 1.2 \mathrm{~mm}$, 
VWR) then left to air dry. Haematoxylin and eosin (H\&E) staining was used to stain vessels by washing the slides in running tap water for 5 mins before placing them in Mayer's Haematoxylin for 10mins. The samples were then washed again in running tap water before washing them in Scott's tap water for 2 mins to stain the nuclear chromatin and nuclear membranes blue. The samples were washed again in running water for 5 mins before placing them in $1 \%$ eosin for 3 mins. For immunohistochemistry, additional $5 \mu \mathrm{m}$ thick sections were blocked firstly with $0.3 \% \mathrm{H}_{2} \mathrm{O}_{2}$ for 20mins. After a 5min PBS wash, 20\% horse serum in PBS was applied for 30mins to block non specific antibody binding. Slides were then washed again for 5 mins in PBS followed by incubation of 1:50 dilution of primary $\alpha$-actin antibody (DakoCytomation) of each sample for $2 \mathrm{hrs}$ at room temperature without shaking. Slides were again washed in PBS for $5 \mathrm{mins}$, before the staining was developed using the avidin-biotin Vectorstain Elite Kit (Universal, Vector laboratories) and antigen localised using 3,3' diaminobenzidine following the manufacturer's instructions. After a brief wash in running water, slides were dehydrated through an ascending series $(70 \%, 90 \%$ and $100 \%)$ of alcohol concentrations for 2 mins each. The samples were then cleared using xylene for 5 mins before mounting the slides with glass coverslips coated in DPX mounting medium before viewing under light microscopy (Zeiss Axiovert 25).

\section{Transmission electron microscopy (TEM)}

Vessel segments from wire myography were fixed overnight with $3 \%$ glutaraldehyde. Following this incubation the tissue specimens were washed in $0.1 \mathrm{M}$ cacodylate buffer. The tissue was then post fixed in $1 \%$ osmium tetroxide in $0.1 \mathrm{M}$ cacodylate buffer. Following five $1 \mathrm{~min}$ washes in distilled water the samples were dehydrated with graded alcohol treatments $(50 \%, 70 \%, 90 \%$ and $100 \%$ for 15 mins each) before finally being treated with $100 \%$ propylene oxide for a final 15 mins. The samples were then infiltrated with resin (mixed with propylene oxide at ratio of 3:1) for $4 \mathrm{~h}$ at RT. Finally, the tissue was embedded in a plastic mould which was left to polymerise overnight. For TEM, ultrathin (70nm) sections were cut using a diamond knife (Diatome) and collected on a copper mesh grid 
ready for viewing using a FEI Tecnai 12 BioTWIN microscope. Images were captured with a Megaview III camera using Soft Imaging System software.

\section{Data analysis}

Recorded wire myography data was converted from tension to active effective pressure (AEP) to take varying vessel sizes into consideration. AEP was calculated using Laplace's equation, dividing recorded active tension $(\mathrm{mN} / \mathrm{mm})$ measurements by the internal vessel radius $(\mathrm{mm})$. Effects are expressed in AEP as a percentage of the maximal AEP achieved with a preceding reference contraction to $10^{-6} \mathrm{M}$ U46619. Where possible, a nonlinear curve fit was performed using Prism 6 (GraphPad Software, La Jolla, USA) to determine the $\mathrm{EC}_{50}$ or $\mathrm{IC}_{50}$ of a concentration-response curve. The three-parameter logistic equation was used to fit all curves, as recommended for data sets with low numbers of data points. Curve fitting was not performed on concentration-response curves lacking recognisable bottom or top plateaus. These incomplete concentration-response curves were caused by limited availability of drugs. To enable the analysis of incomplete concentration-response curves, responses to drug and vehicle were compared using a mixed two-way ANOVA reporting $\mathrm{p}$ values for the treatment factor. The null hypothesis was rejected at $p<0.05$. Graphs show mean and SEM unless stated otherwise.

\section{Results}

\section{Wire myography}

U46619, as a well-known vasoconstrictor in placental vessels, caused the strongest contraction of all tested agents with an $\mathrm{EC}_{50}$ of $1.2 * 10^{-7} \mathrm{M}$. The contraction gave stable plateaus at each concentration point and reversed to baseline within $1 \mathrm{~h}$ of starting PSS washes. Phenylephrine and noradrenaline were tested as well-known vasoconstrictors of the systemic vasculature. Only phenylephrine caused a small statistically significant contraction at high concentrations $\left(3.3^{*} 10^{-5} \mathrm{M}\right)$. Arginine vasopressin 
169 (AVP) did not show any effect in stem villous arteries. Results for U46619, phenylephrine, noradrenaline and AVP are shown in Figure 1.

Figure 2 shows results for 5-HT, angiotensin II and endothelin-1. 5-HT resulted in a contraction of stem villous arteries with an $\mathrm{EC}_{50}$ of $1.1^{*} 10^{-7} \mathrm{M}$, but the maximum $\mathrm{AEP}$ was only about a fifth of U46619's effect. Angiotensin II caused small initial contractions that were not sustained and not consistent across tested vessels. The concentration-response curve therefore did not depict any significant effect of this compound. Endothelin-1 caused the second strongest contraction of tested compounds in stem villous arteries with about $60 \%$ of U46619's AEP at $10^{-6} \mathrm{M}$. Due to limited availability of drug, it was not possible to test higher endothelin-1 concentrations for the determination of relative $R_{\max }$ to U46619. The contractions to endothelin-1 resulted in stable plateaus that were difficult to wash out. Endothelin-1 induced contractions did not return to the initial baseline within $2 \mathrm{~h}$, even after numerous washes using PSS.

Results for test compounds examined for relaxant properties are shown in Figure 3 and Figure 4. Acetylcholine, bradykinin and substance P did not cause any effects in stem villous arteries. Histamine and sodium nitroprusside (SNP) relaxed vessels to about $50 \%$ of the preconstricted AEP with $\mathrm{IC}_{50}$ of $1.7^{*} 10^{-6} \mathrm{M}$ and $7 * 10^{-6} \mathrm{M}$ respectively. SQ20548 was the most potent relaxant amongst the test compounds, relaxing the vessel back to baseline tensions with an $I C_{50}$ of $2.3^{*} 10^{-7} \mathrm{M}$. Other substances that showed significant effects were riluzole, nifedipine and papaverine. All vessels relaxed back to baseline levels when drugs were washed out using SPSS.

\section{IHC and TEM}

IHC and TEM imaging of the vessel segments enabled a detailed examination of the cell layers within stem villous arteries after wire myography experiments. It was of special interest to verify the integrity of the endothelial layer in order to interpret the effects seen with wire myography. Figure 5 depicts a subsection of a stem villus showing the three important portions of a stem villous artery; namely the lumen, EC and SMC are present. A stem villous artery and vein typically run in close 
proximity to each other within one stem villous branch. In Figure 6, the single cell layer of endothelial cells (EC) can be distinguished by the elastic lamina (EL) which separates the EC layer from smooth muscle cells (SMC). Figure 5 and Figure 6 show that the EC layer does appear to remain intact following vessel isolation and myography.

\section{Discussion}

The placental circulation facilitates adequate supply of nutrients and gases to the fetus. Still little is known about the physiological behaviour of placental resistance vessels and their role in pregnancy complications. For this reason, the present study aimed to evaluate the effect of a range of pharmacological compounds and endogenous lipids on human placental arteries.

\section{Effects of various pharmacological compound on stem villus arteries}

Given the potential significance of stem villous arteries to placental dysfunction, a selection of pharmacological compounds was assessed for their potential contractile or relaxant effects. Two well-known constrictors of the placental vasculature, thromboxane agonist U46619 and endothelin1 caused reliable and strong contractions in stem villous arteries as previously demonstrated [7-9, 14]. The stable thromboxane $A_{2}$ receptor agonist $U 46619$ is a strong and reliable vasoconstrictor. This property makes it a commonly used tool to assess vascular function in uteroplacental vessels. It has been shown by a number of groups that maximum tension development in response to U46619 is significantly lower in pre-eclampsia, whereas there is no difference in the sensitivity. This has been shown for stem villous arteries [14], chorionic plate arteries $[15,16]$ and in a perfusion model of placental lobules[17].

AVP as a reliable vasoconstrictor of the systemic vasculature did not cause any contraction in stem villous arteries. Vasoconstriction in response to AVP was reported in chorionic plate arteries, but stem villous arteries seem to be inert against this substance $[10,11,15,16]$. This may be explained 
by a low placental expression of the AVP receptor, 1A (AVPR1A), which is the main subtype involved in AVP's contractile effect [18].

5-HT concentration-response curves showed mild contractions in stem villous arteries, which is in line with previous findings $[7,11]$ supporting observations for the presence of $5-\mathrm{HT}$ receptors in the placenta [19].

Angiotensin II caused transient contractions in stem villous arteries that were prone to tachyphylaxis as previously noted by others $[11,20,21]$. There are also reports of sustained angiotensin-II contractions, but exclusively in chorionic plate arteries or perfused placental lobule preparations [1, 22, 23]. Tachyphylaxis to angiotensin II is documented for many tissues other than placentae and is thought to be caused by internalisation or allosteric conformational change of the angiotensin II receptor [24].

As the placenta lacks autonomic innervation, it was not unexpected that substances of the autonomic nervous system showed little or no effect [2]. While noradrenaline did not affect vessel tension at all, phenylephrine caused a weak contraction at high concentrations $\left(3.3^{*} 10^{-5} \mathrm{M}\right)$. Despite its importance in the systemic vasculature, previous reports indicate that noradrenaline has reduced effects on placental vessels. No effects of noradrenaline could be observed in stem villous arteries, chorionic plate arteries or placental lobules $[10,11,25]$. In the case of phenylephrine, transient and unreliable contractions of chorionic plate veins were reported, which is similar to the effects seen in stem villous arteries in the present study $[10,26]$.

Similarly, as for previously discussed contractile agents, the lack of autonomic innervation can be observed in the ineffectiveness of several known relaxant agents. Acetylcholine (ACh) did not cause any relaxation of preimposed tone although it is a strong vasodilator in the systemic vasculature. The findings of the present study are supported by the observation that the cholinergic agonist, carbachol, did not show any effects in preconstricted chorionic plate arteries [10]. ACh was previously demonstrated to be endogenously released from single placental cotyledons and whole placentae [27]. Protein and mRNA expression of the nicotinic ACh receptor were demonstrated in 
the human placental vasculature, while the muscarinic ACh receptor could only be detected in syncytiotrophoblasts but not in placental vessels $[28,29]$. ACh could therefore potentially act on the placental vasculature via these $\mathrm{ACh}$ receptors and currently there is no evidence to explain its lack of impact on vascular tone.

Two other endothelium-dependent vasodilators, bradykinin and substance $P$, similarly did not cause any alteration of the preimposed tone in stem villous arteries. This is again in line with findings of a range of authors who worked with chorionic plate arteries and stem villous arteries $[10,12,16,30]$. Bradykinin has frequently been used as endothelium-dependent vasodilator in studies on uteroplacental blood vessels, via release of NO, prostacyclin and EDHF. The endothelium-dependent vasodilator substance $\mathrm{P}$ is a peptide that plays an important role as a neurotransmitter [31].

Of all endothelium-dependent dilators, only histamine gave reliable relaxation to preimposed tone. Previous work observed a relaxation to histamine in stem villous arteries, which could only be seen in vessels that were not denuded of endothelium [7]. This supports that the endothelium in examined stem villous arteries of this study was intact, as also shown by TEM and IHC imaging. In contrast to this, a number of authors reported contractile instead of relaxant effects of histamine in chorionic plate arteries [32-34]. It was later found in chorionic plate arteries that part of the histamine induced relaxation is regulated via the H1-receptor mediated endothelium-dependent pathway and part by a direct $\mathrm{H} 2$-receptor mediated VSMC relaxation [35]. An initial contractile element at low concentrations of the histamine dose response was achieved over a direct $\mathrm{H2}$ receptor mediated VSMC activation. In the present study, no contractile element was noted in the histamine dose response, which could indicate a different mechanism of action of histamine in stem villous arteries compared to chorionic plate arteries. However, the preconstruction of vessels in this study might have masked a contractile element of the histamine effect, hence a more detailed investigation is needed to confirm the behaviour of stem villous arteries to histamine.

The strongest relaxing effect of all tested substances was observed for SQ29548. The thromboxane $\mathrm{A}_{2}$ receptor antagonist was shown to reduce the sensitivity to U-46619 and 8-isoPGE 2 induced 
contractions in chorionic plate arteries and a placental lobule perfusion model [36, 37]. The relaxation back to baseline levels is not unexpected, as vessels were precontracted using thromboxane receptor agonist U46619. SQ29548 is most probably acting as a competitive receptor antagonist to U46619.

Another strong vasodilator of stem villous arteries was sodium nitroprusside (SNP), which emphasises the important role of NO for the control of the placental vasculature. This is consistent with previous reports in stem villous arteries [7, 9] and chorionic plate arteries [10, 38]. Other tested endothelium-independent dilators were riluzole and papaverine. Both caused relaxation of the preimposed tone. Papaverine was previously shown to relax chorionic plate arteries [10] and riluzole was shown to relax stem villous arteries and chorionic plate arteries . The endothelium-independent blood vessel relaxant papaverine was first isolated from opium and acts as a PDE inhibitor and calcium channel modulator. The compound riluzole is a glutamate antagonist, sodium channel blocker and potassium channel opener, used for treatment of amyotrophic lateral sclerosis [39]. It acts on TREK-1 (a two-pore-domain potassium channel), which is expressed in placental vessels.

As important drug for the treatment of non-gestational and gestational hypertension, the vascular effects of calcium channel antagonist nifedipine were examined. The compound caused relaxation of the preimposed tone in stem villous arteries. Relaxant effects of nifedipine or nitrendipine were previously demonstrated in chorionic plate arteries [40-42] and stem villous arteries [21]. This indicates the presence of L-type calcium channels in stem villous arteries, which were previously only demonstrated to be expressed in trophoblasts [43].

In summary, stem villous arteries responded to a wide profile of pharmacological compounds. Contractile effects in stem villous arteries were caused by U46619, 5-HT, angiotensin II and endothelin-1, whereas noradrenaline and AVP failed to result in a contraction. Dilating effects were seen for histamine, riluzole, nifedipine, papaverine, SNP and SQ29548 but not for acetylcholine, 
bradykinin and substance P. These findings were mostly consistent with research conducted in placental vessels as reviewed above.

In general, it is observed that commonly used vasoactive substances of the systemic vasculature such as noradrenaline, AVP and acetylcholine seem to hardly affect stem villous arteries. This is a common finding in all placental vessels and attributable to the missing innervation in the placenta [2].

Placental vessels clearly behave differently to vessels of the systemic vasculature. Chorionic plate arteries and stem villous arteries show similar behaviour in many cases but there are several exemptions as well: AVP did not affect stem villous arteries whereas a contraction in chorionic plate arteries was reported by several authors $[10,15,44,45]$. Furthermore, no contractile effect of histamine could be observed, as reported in chorionic plate arteries [32-34]. Given their importance in the placental circulation, it is therefore important to consider stem villous arteries as a distinct vascular bed in future research.

\section{The endothelium in the placental vasculature}

The integrity of the endothelium in the experimental setup is of particular interest, as effects various compounds are dependent on its presence. Therefore, experimental protocols typically involve checking endothelial function using acetylcholine [3]. However, acetylcholine and other endothelium-dependent vasodilators as bradykinin and substance $\mathrm{P}$ did not affect vascular tension in stem villous arteries as previously shown by a number of authors $[6,7,10,12,16,30]$. An evaluation of endothelial integrity in the present study using the conventional acetylcholine relaxation was therefore not possible. Only one endothelium-dependent dilator, histamine, caused vasorelaxation whereby part of the dilating effect is, at least in chorionic plate arteries, attributed to an endothelium-independent process [35]. Assessment of the endothelial function in stem villous arteries revealed that histamine induced relaxations only in presence of the endothelium $[7,8]$. These relaxations to histamine were also observed in the present study, which is an indicator that 
the endothelium of stem villous arteries used in this study was intact. However, the role of the endothelium in stem villous arteries is poorly characterised. It is also doubtful that knowledge from other vascular beds such as chorionic plate arteries can be transferred and applied to stem villous arteries as they show considerably different vascular behaviour. For this reason, an in depth investigation is required to evaluate the effect of endothelium removal on vascular function in stem villous arteries. TEM imaging showed that the endothelium of stem villous arteries is present after the mounting procedure. The discrepancy of the endothelial function when comparing to other vascular beds can therefore only be explained on cellular level. The absence of effects caused by bradykinin or acetylcholine could also be explained by elevated intrinsic NO levels in the pregnancy [6]. Permanent basal NO production is thought to be key for the physiological maintenance of low vascular resistance in the placenta $[1,5,6]$. At the same time, NO inhibits CYP enzymes and with that the release of EDHF [46]. There are various compounds produced by CYP that are thought to contribute to the EDHF effect [47]. In general, it was suggested that the EDHF pathway might act as backup mechanism in vessels with impaired NO availability possibility due to endothelial dysfunction [48]. In the experimental setup of the present study, NO release by bradykinin or acetylcholine might not considerably add to the already increased NO availability. Furthermore, the NO-independent, CYP dependent component of the bradykinin/acetylcholine relaxation might be attenuated as CYP enzymes are blocked by high NO levels. However, this hypothesis needs to be tested and confirmed.

In conclusion, the assessment of various pharmacological compounds provided a valuable overview of the physiological behaviour of stem villous arteries. This work will also be useful knowledge for future studies, where pharmacological tools are required to assess vascular function. Substances that are part of the autonomous system such as noradrenaline or acetylcholine showed no effects in stem villous arteries, which cuts the placenta off from the systemic maternal vascular regulation. The fact that stem villous arteries responded to a range of mediators that were previously reported to elicit altered vascular effects in pre-eclampsia, creates the base for future research on stem 
349 villous arteries in the context of hypertensive gestational diseases. In this context, use of more specific blockers targeting individual pathways would enable a detailed understanding of the placental physiology.

Our observation that particularly endothelium-dependent processes were attenuated in the placental vasculature indicates that there is an urgent need to determine the role of the endothelium in the placenta in future studies.

355

\section{Acknowledgements}

357 We thank the patients for participating in this study and the clinical staff of the Department of Obstetrics and Gynaecology at the Royal Derby Hospital for their cooperation. This work was supported by the British Heart Foundation [grant number: PG/10/49/28422] and the University of Nottingham (studentship).

\section{Conflict of interest}

None.

\section{References}

[1] W.A. Walters, A.L. Boura, Regulation of fetal vascular tone in the human placenta, Reprod Fertil Dev 3(4) (1991) 475-481.

[2] F.D. Reilly, P.T. Russell, Neurohistochemical evidence supporting an absence of adrenergic and 
[4] R. Demir, G. Kosanke, G. Kohnen, S. Kertschanska, P. Kaufmann, Classification of human placental stem villi: review of structural and functional aspects, Microsc Res Tech 38(1-2) (1997) 29-41.

[5] L. Poston, The control of blood flow to the placenta, Exp Physiol 82(2) (1997) 377-387.

[6] S.M. Sladek, R.R. Magness, K.P. Conrad, Nitric oxide and pregnancy, Am J Physiol 272(2 Pt 2) (1997) R441-R463.

[7] S. Sabry, F. Mondon, F. Ferré, A.T. Dinh-Xuan, In vitro contractile and relaxant responses of human resistance placental stem villi arteries of healthy parturients: role of endothelium, Fundam Clin Pharmacol 9(1) (1995) 46-51.

[8] S. Sabry, F. Mondon, M. Levy, F. Ferré, A.T. Dinh-Xuan, Endothelial modulation of vasoconstrictor responses to endothelin-1 in human placental stem villi small arteries, Br J Pharmacol 115(6) (1995) 1038-1042.

[9] H.V. Clausen, J.C. Jorgensen, B. Ottesen, Stem villous arteries from the placentas of heavy smokers: functional and mechanical properties, Am J Obstet Gynecol 180(2 Pt 1) (1999) 476-482.

[10] M. Wareing, I.P. Crocker, A.Y. Warren, M.J. Taggart, P.N. Baker, Characterization of small arteries isolated from the human placental chorionic plate, Placenta 23(5) (2002) 400-409.

[11] J. Allen, A. Forman, S. Maigaard, L.T. Jespersen, K.E. Andersson, Effect of endogenous vasoconstrictors on maternal intramyometrial and fetal stem villous arteries in pre-eclampsia, J Hypertens 7(7) (1989) 529-536.

[12] V. Hansen, S. Maigaard, J. Allen, A. Forman, Effects of vasoactive intestinal polypeptide and substance P on human intramyometrial arteries and stem villous arteries in term pregnancy, Placenta 9(5) (1988) 501-506. 
393

394

395

396

397

398

399

400

401

402

403

404

405

406

407

408

409

410

411

412

413

414

[13] M. Wareing, S.L. Greenwood, P.N. Baker, Reactivity of human placental chorionic plate vessels is modified by level of oxygenation: differences between arteries and veins, Placenta 27(1) (2006) 4248.

[14] S. Jerat, D.W. Morrish, S.T. Davidge, S. Kaufman, Effect of adrenomedullin on placental arteries in normal and preeclamptic pregnancies, Hypertension 37(2) (2001) 227-231.

[15] M. Wareing, P.N. Baker, Vasoconstriction of small arteries isolated from the human placental chorionic plate in normal and compromised pregnancy, Hypertens Pregnancy 23(3) (2004) 237-246.

[16] M. Wareing, J.E. Myers, M. O'Hara, L.C. Kenny, M.J. Taggart, L. Skillern, I. Machin, P.N. Baker, Phosphodiesterase- 5 inhibitors and omental and placental small artery function in normal pregnancy and pre-eclampsia, Eur J Obstet Gynecol Reprod Biol 127(1) (2006) 41-49.

[17] M.A. Read, I.M. Leitch, W.B. Giles, A.M. Bisits, A.L. Boura, W.A. Walters, U46619-mediated vasoconstriction of the fetal placental vasculature in vitro in normal and hypertensive pregnancies, J Hypertens 17(3) (1999) 389-396.

[18] M. Thibonnier, M.K. Graves, M.S. Wagner, C. Auzan, E. Clauser, H.F. Willard, Structure, sequence, expression, and chromosomal localization of the human V1a vasopressin receptor gene, Genomics 31(3) (1996) 327-334.

[19] W.Q. Huang, C.L. Zhang, X.Y. Di, L. Sun, Microscopic and ultramicroscopic localizations and quantitative analysis of 5-HT receptors in human placentas, Chinese Science Bulletin 43(10) (1998) 804-809.

[20] S. Maigaard, A. Forman, K.E. Andersson, Differential effects of angiotensin, vasopressin and oxytocin on various smooth muscle tissues within the human uteroplacental unit, Acta Physiol Scand 128(1) (1986) 23-31. 
415

416

417

418

419

420

421

422

423

424

425

426

427

428

429

430

431

432

433

434

435

[21] J. Allen, K. Skajaa, S. Maigaard, A. Forman, Effects of vasodilators on isolated human uteroplacental arteries, Obstet Gynecol 77(5) (1991) 765-771.

[22] C.U. Odum, F. Broughton Pipkin, Studies on the effects of angiotensin II on human chorionic plate arteries and their modification by a calcium antagonist, nitrendipine, $\mathrm{Br} J$ Clin Pharmacol 24(1) (1987) 15-19.

[23] C.U. Odum, F. Broughton Pipkin, Studies on the response of isolated human chorionic plate artery strips to angiotensin II in normal pregnancy and in pregnancy induced hypertension, West Afr J Med 8(4) (1989) 251-256.

[24] C.A. Kanashiro, T.B. Paiva, A.C. Paiva, R.N. Prioste, J. Aboulafia, S.I. Shimuta, Angiotensin II tachyphylaxis in the guinea pig ileum and its prevention: a pharmacological and biochemical study, J Pharmacol Exp Ther 275(3) (1995) 1543-1550.

[25] A. Inayatulla, S. Chemtob, B. Nuwayhid, D.R. Varma, Responses of placental arteries from normotensive and preeclamptic women to endogenous vasoactive agents, Am J Obstet Gynecol 168(3 Pt 1) (1993) 869-874.

[26] M. Wareing, S.L. Greenwood, M.J. Taggart, P.N. Baker, Vasoactive responses of veins isolated from the human placental chorionic plate, Placenta 24(7) (2003) 790-796.

[27] B.V. Rama Sastry, J. Olubadewo, R.D. Harbison, D.E. Schmidt, Human placental cholinergic system. Occurrence, distribution and variation with gestational age of acetylcholine in human placenta, Biochem Pharmacol 25(4) (1976) 425-431.

[28] K.S. Lips, D. Brüggmann, U. Pfeil, R. Vollerthun, S.A. Grando, W. Kummer, Nicotinic acetylcholine receptors in rat and human placenta, Placenta 26(10) (2005) 735-746. 
[29] S.K. Tayebati, L. Vitaioli, D. Zaccheo, F. Amenta, Autoradiographic localisation of muscarinic cholinergic receptor subtypes in human placenta, Neurosci Lett 247(2-3) (1998) 167-170.

[30] S.S. Ong, R.J. Moore, A.Y. Warren, I.P. Crocker, J. Fulford, D.J. Tyler, P.A. Gowland, P.N. Baker, Myometrial and placental artery reactivity alone cannot explain reduced placental perfusion in preeclampsia and intrauterine growth restriction, BJOG 110(10) (2003) 909-915.

[31] C. Bossaller, K. Reither, C. Hehlert-Friedrich, W. Auch-Schwelk, K. Graf, M. Gräfe, E. Fleck, In vivo measurement of endothelium-dependent vasodilation with substance P in man, Herz 17(5) (1992) 284-290.

[32] J. Reviriego, M.S. Fernandez-Alfonso, J. Marín, Actions of vasoactive drugs on human placental vascular smooth muscle, Gen Pharmacol 21(5) (1990) 719-727.

[33] C.W. Quist, R. Vasan, E. Quist, Mechanisms of prostaglandin F2 alpha and histamine-induced contractions in human chorionic vasculature, J Cardiovasc Pharmacol 28(3) (1996) 363-370.

[34] C. Bertrand, J. St-Louis, Reactivities to serotonin and histamine in umbilical and placental vessels during the third trimester after normotensive pregnancies and pregnancies complicated by preeclampsia, Am J Obstet Gynecol 180(3 Pt 1) (1999) 650-659.

[35] T.A. Mills, M.J. Taggart, S.L. Greenwood, P.N. Baker, M. Wareing, Histamine-induced contraction and relaxation of placental chorionic plate arteries, Placenta 28(11-12) (2007) 1158-1164.

[36] M.L. Ogletree, D.N. Harris, R. Greenberg, M.F. Haslanger, M. Nakane, Pharmacological actions of SQ 29,548, a novel selective thromboxane antagonist, J Pharmacol Exp Ther 234(2) (1985) 435-441.

[37] L. Hausermann, J. St-Louis, Thromboxane and isoprostane share the same prostanoid receptors to increase human placental tone, Placenta 32(12) (2011) 941-948. 
[38] C. González, M.A. Cruz, V. Gallardo, P. Miguel, G. Carrasco, Relative potency of nitrovasodilators on human placental vessels from normal and preeclamptic pregnancies, Gynecol Obstet Invest 43(4) (1997) 219-224.

[39] A. Cadaveira-Mosquera, S.J. Ribeiro, A. Reboreda, M. Pérez, J.A. Lamas, Activation of TREK currents by the neuroprotective agent riluzole in mouse sympathetic neurons, J Neurosci 31(4) (2011) 1375-1385.

[40] S. Maigaard, A. Forman, K.E. Andersson, Effects of nifedipine on human placental arteries, Gynecol Obstet Invest 18(4) (1984) 217-224.

[41] H. Kook, Y.D. Yoon, Y.H. Baik, Effects of calcium antagonists on contractions of chorionic arteries in normal and preeclampsia placenta, J Korean Med Sci 11(3) (1996) 250-257.

[42] R. David, I.M. Leitch, M.A. Read, A.L. Boura, W.A. Walters, Actions of magnesium, nifedipine and clonidine on the fetal vasculature of the human placenta, Aust N Z J Obstet Gynaecol 36(3) (1996) 267-271.

[43] R. Moreau, A. Hamel, G. Daoud, L. Simoneau, J. Lafond, Expression of calcium channels along 1473-1479.

[44] S. Maigaard, A. Forman, K.E. Andersson, Relaxant and contractile effects of some amines and prostanoids in myometrial and vascular smooth muscle within the human uteroplacental unit, Acta Physiol Scand 128(1) (1986) 33-40. 
479 [46] J. Bauersachs, R. Popp, M. Hecker, E. Sauer, I. Fleming, R. Busse, Nitric oxide attenuates the

480 release of endothelium-derived hyperpolarizing factor, Circulation 94(12) (1996) 3341-3347.

481 [47] W.B. Campbell, D. Gebremedhin, P.F. Pratt, D.R. Harder, Identification of epoxyeicosatrienoic

482 acids as endothelium-derived hyperpolarizing factors, Circ Res 78(3) (1996) 415-423.

483 [48] I. Fleming, Cytochrome p450 and vascular homeostasis, Circ Res 89(9) (2001) 753-762.

484 [49] J. Gardosi, A. Chang, B. Kalyan, D. Sahota, E.M. Symonds, Customised antenatal growth charts,

485 Lancet 339(8788) (1992) 283-287.

486 [50] J. Gardosi, M. Mongelli, M. Wilcox, A. Chang, An adjustable fetal weight standard, Ultrasound 487 Obstet Gynecol 6(3) (1995) 168-174. 


\section{A}

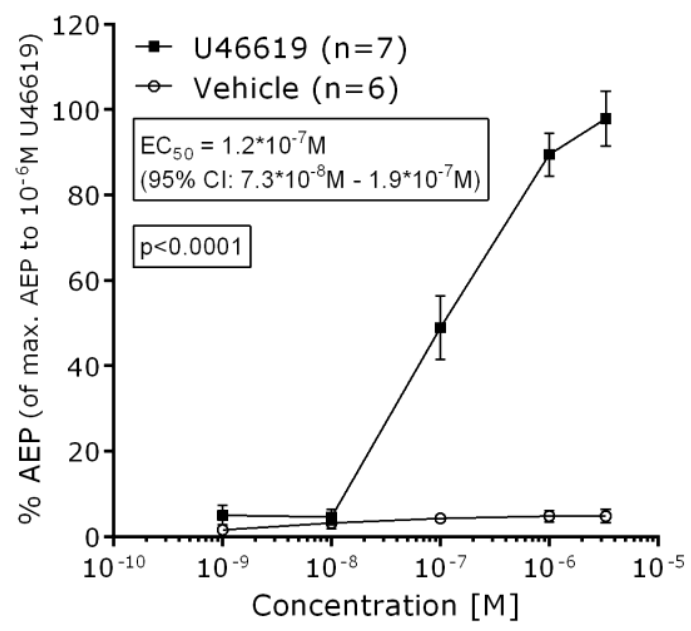

\section{C}

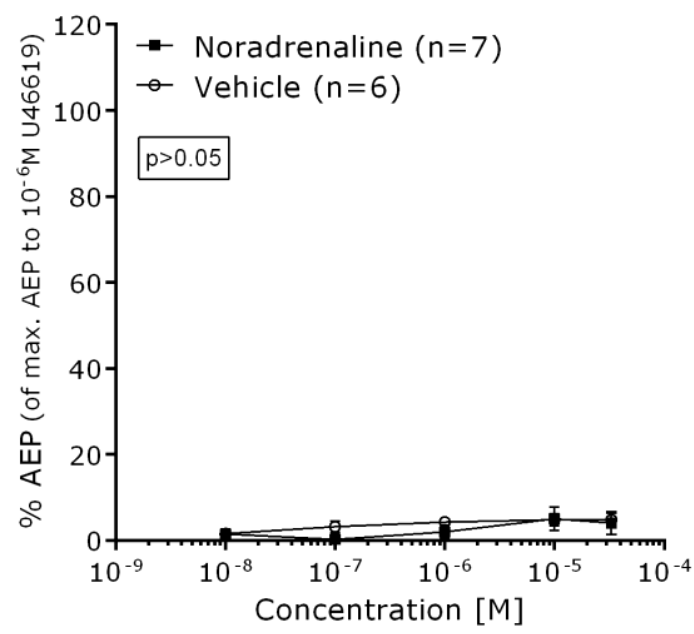

B

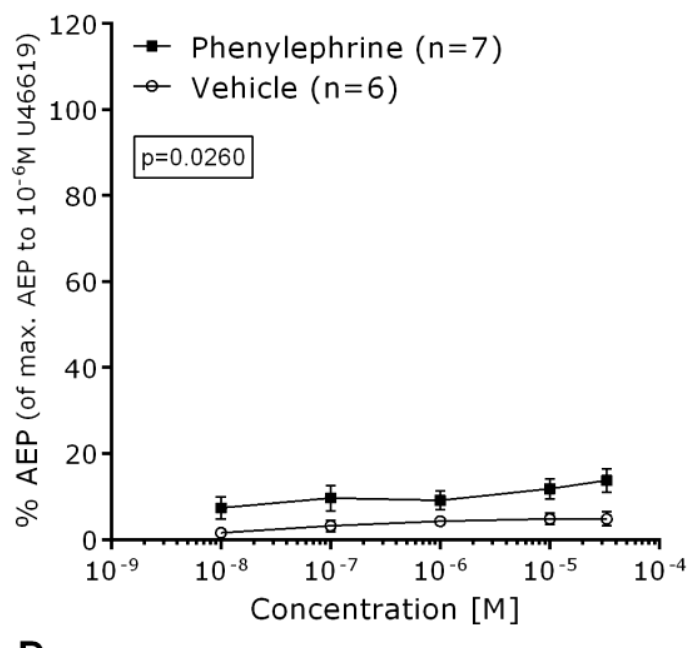

D

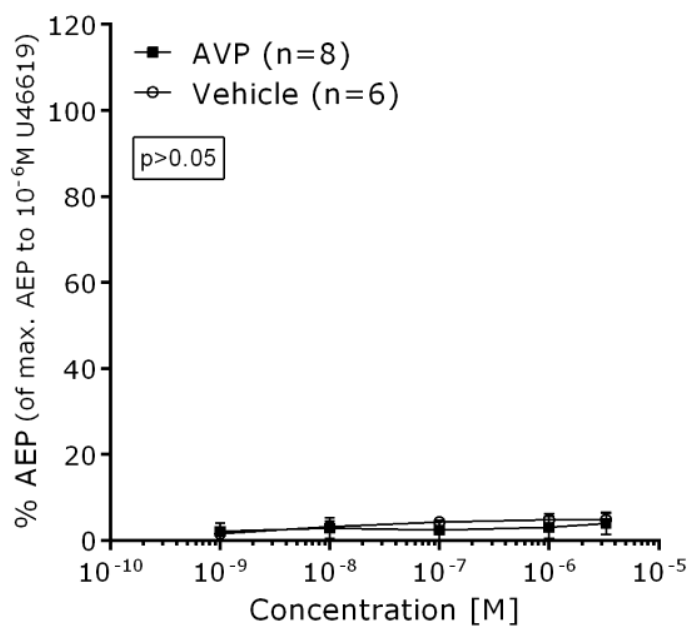

489

490 Figure 1: Effect of (A) U46619, (B) phenylephrine, (C) noradrenaline and (D) AVP on stem villous arteries. Bars show

491 mean and SEM with solid squares representing the tested substance and open circles representing the vehicle control.

492 Effects are expressed as AEP in percent of the maximal AEP achieved with a preceding reference contraction to $10^{-6} \mathrm{M}$ of 
A

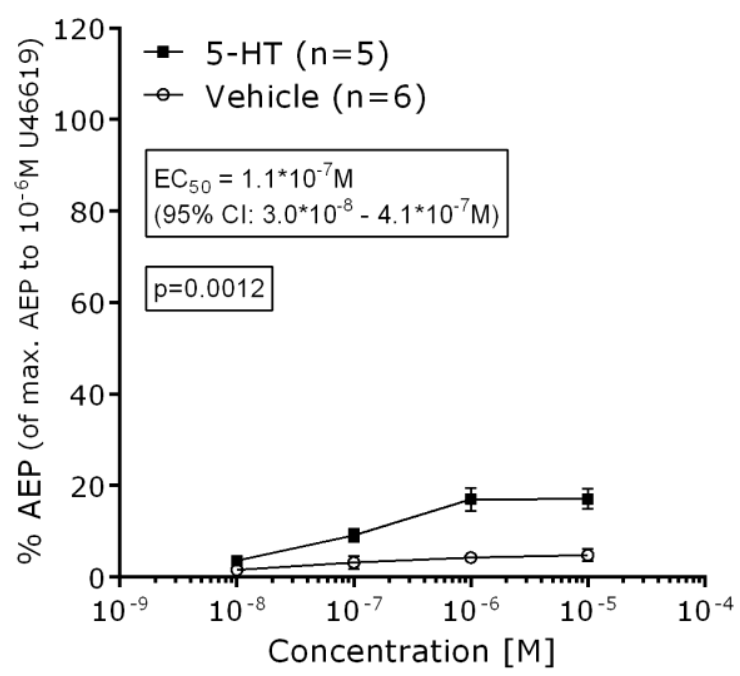

C

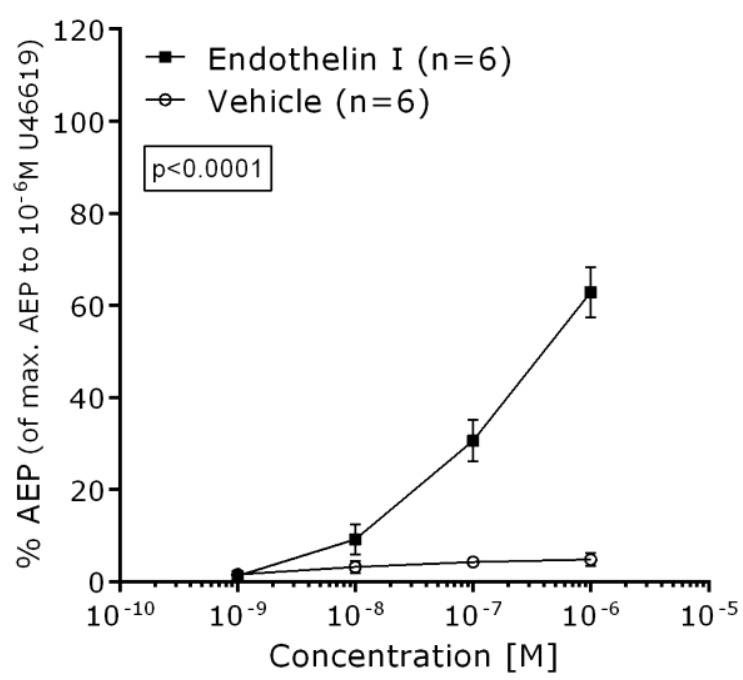

B

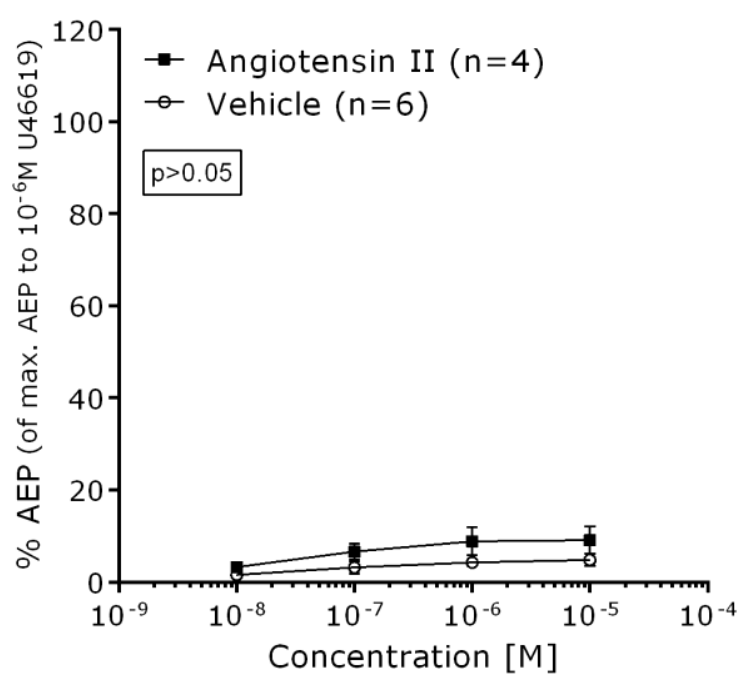

494

495 Figure 2: Effect of (A) 5-HT, (B) angiotensin II and (C) endothelin-1 on stem villous arteries. Bars show mean and SEM

496 with solid squares representing the tested substance and open circles representing the vehicle control. Effects are expressed as AEP in percent of the maximal AEP achieved with a preceding reference contraction to $10^{-6} \mathrm{M}$ of $\mathrm{U} 46619$. All vessels were normalised to $0.9 * I C_{5.1} \mathrm{kPa}$. Significance was tested using a mixed two-way ANOVA. 

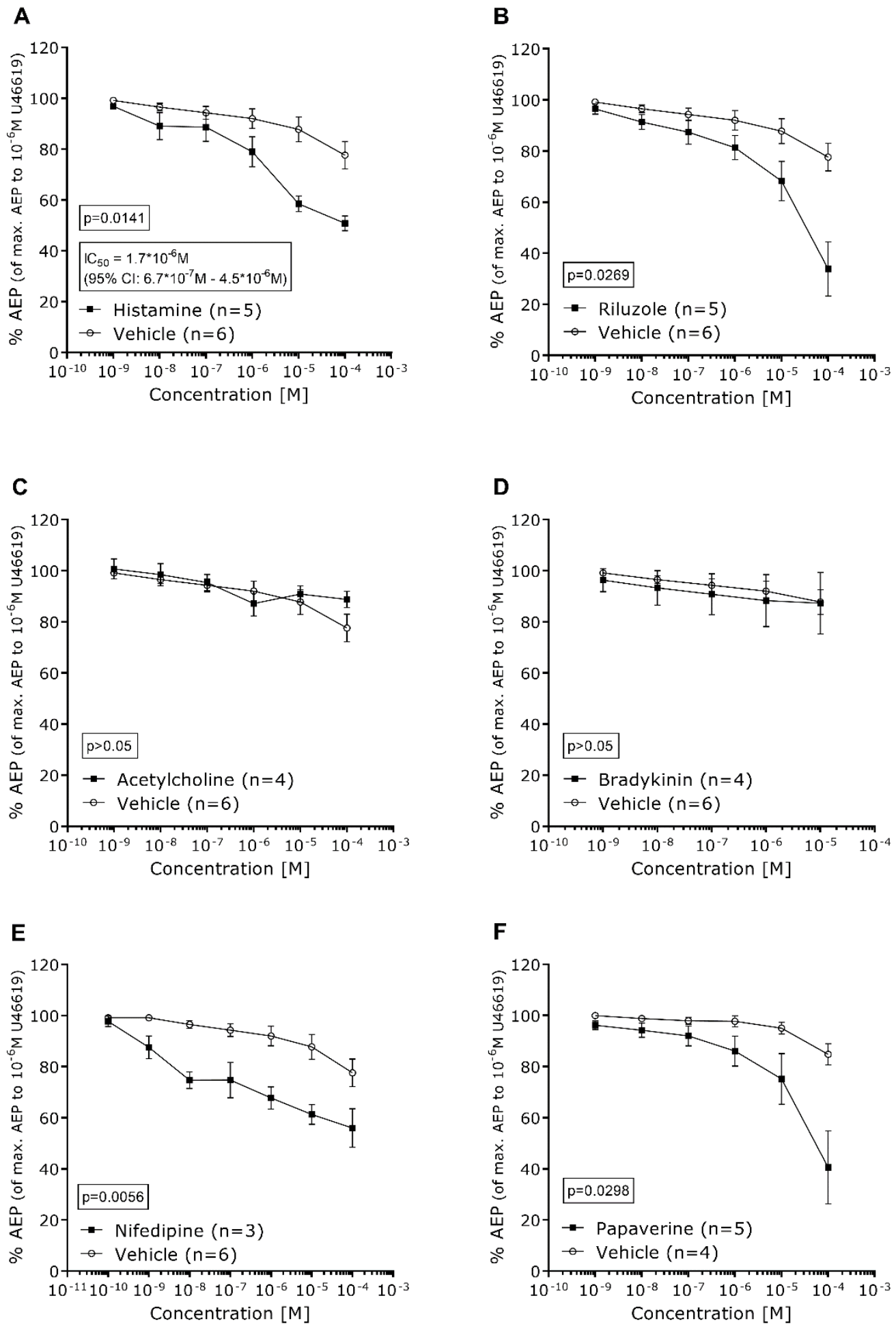

501 Figure 3: Effect of (A) histamine, (B) riluzole, (C) acetylcholine, (D) bradykinin, (E) nifedipine and (F) papaverine on stem

502 villous arteries. Bars show mean and SEM, solid squares representing the tested substance and open circles representing the vehicle control. Effects are expressed as AEP in percent of the maximal AEP achieved with a preceding reference contraction to $10^{-6} \mathrm{M}$ of U46619. All vessels were normalised to $0.9 * I \mathrm{C}_{5.1} \mathrm{kPa}$. Significance was tested using a mixed twoway ANOVA. 
A

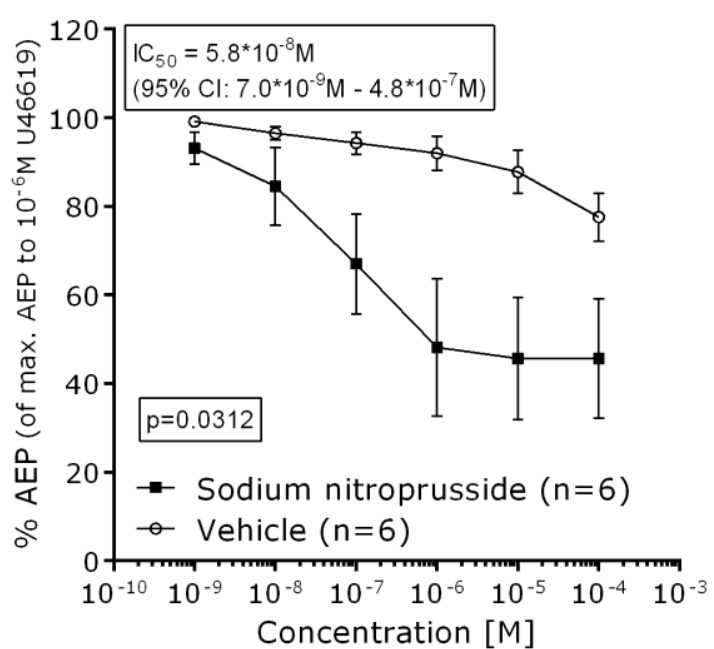

C

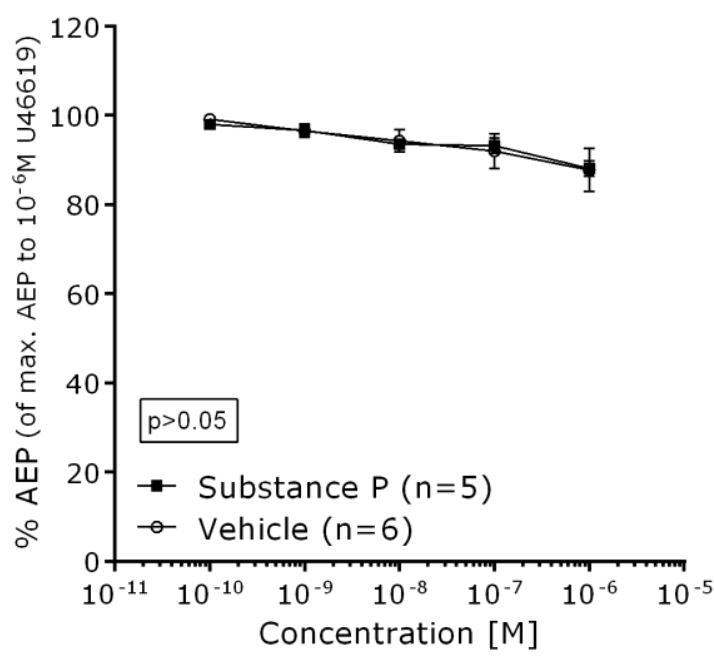

B

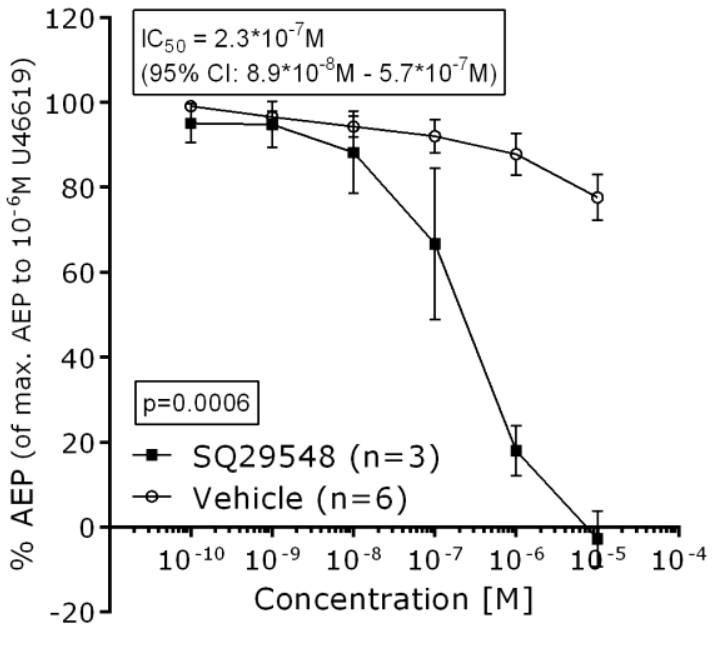

507

508 Figure 4: Effect of (A) sodium nitroprusside, (B) SQ29548 and (C) substance P on stem villous arteries. Bars show mean

509 and SEM with solid squares representing the tested substance and open circles representing the vehicle control. Effects

510 are expressed as AEP in percent of the maximal AEP achieved with a preceding reference contraction to $10^{-6} \mathrm{M}$ of

511 U46619. All vessels were normalised to $0.9 * I C_{5.1} \mathrm{kPa}$. Significance was tested using a mixed two-way ANOVA. 
513

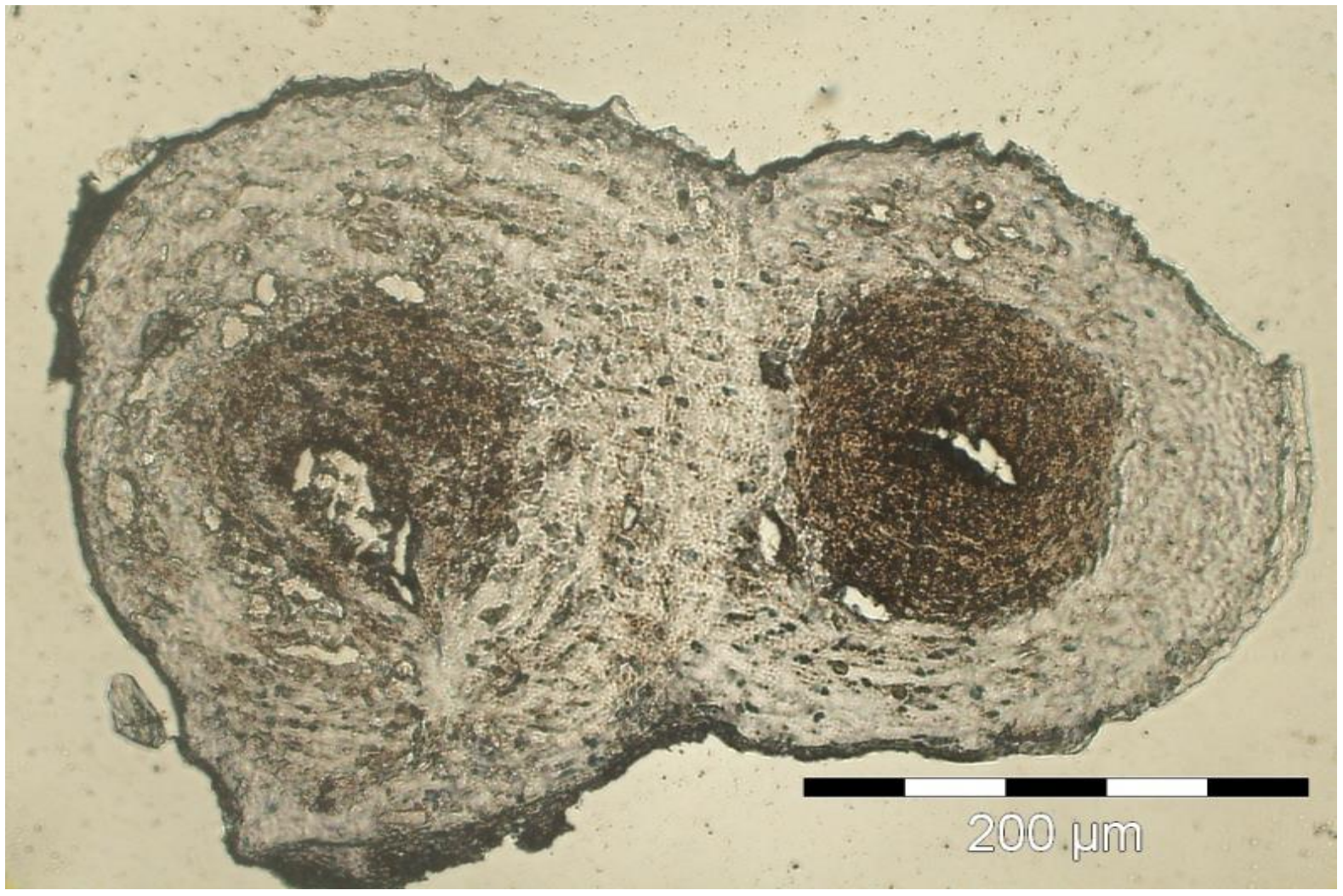

514 Figure 5: IHC showing a subsection of a stem villus. The SMC layer around the artery (right) detected with $\alpha$-actin is

515 thicker and more prominent compared to the vein (left). The endothelium can be seen as a dense stain around the

516 lumen of the stem villous artery. 


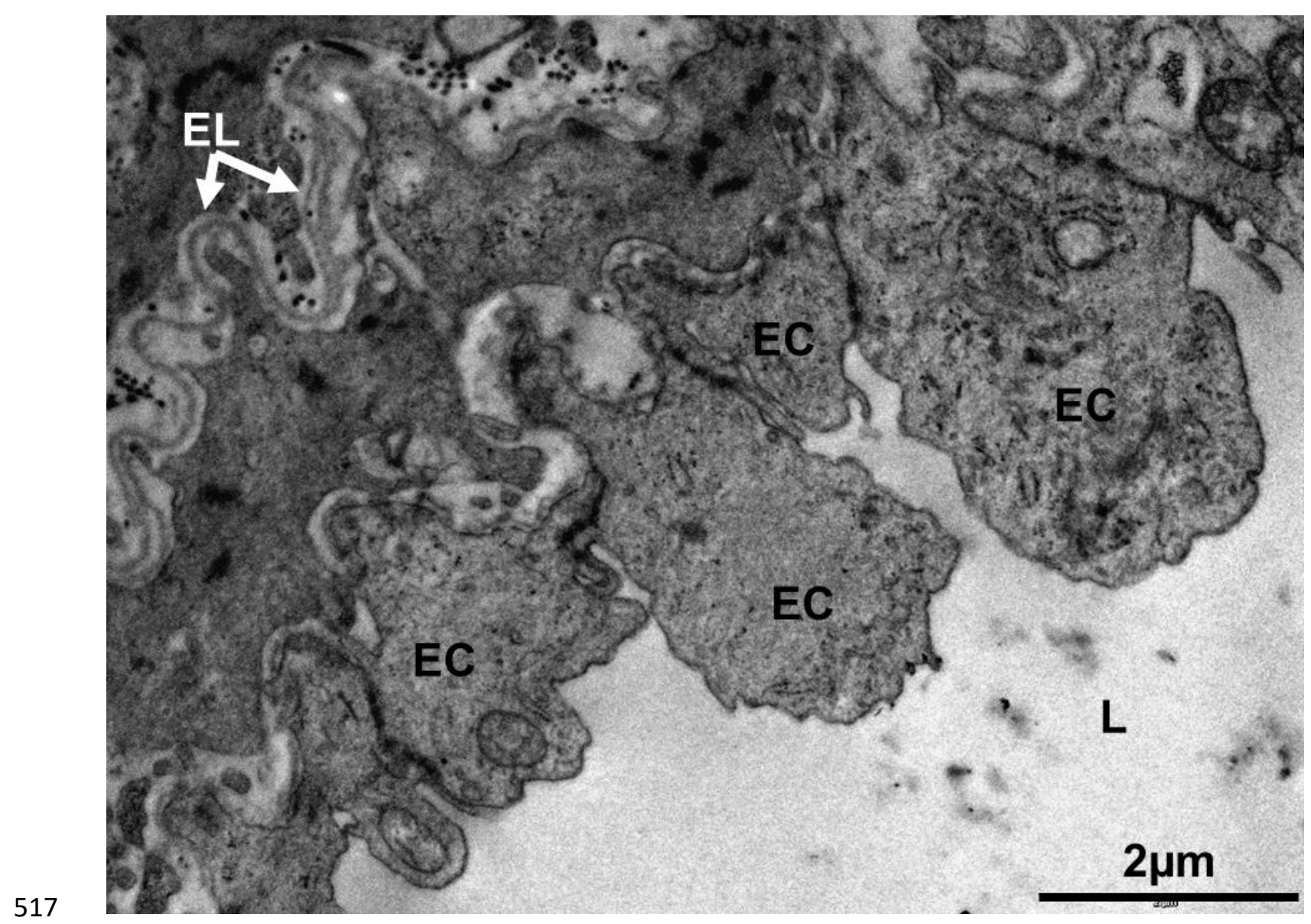

518 Figure 6: TEM showing a subsection of a stem villous artery with intact endothelium. EC: Endothelial cell; EL: Elastic 519 lamina; L: Lumen. 
521 Tables

522

523 Table 1: Patient demographics for collected placentae. Table shows mean (standard deviation) or total numbers. $\mathrm{N}=33$.

524 yrs: years; wks: weeks. Customised weight centiles were calculated using Weight Centile Calculator from GROW

525 software version 8.0.4 (UK), $2019[49,50]$.

\begin{tabular}{ll}
\hline Age [yrs] & $32.1(6.2)$ \\
BMI (at booking) & $30.1(7.9)$ \\
Gravida & $3.1(1.5)$ \\
Parity & $1.4(1.1)$ \\
Gestational week at delivery [wks] & $38.5(1.2)$ \\
Birthweight [g] & $3491.8(548.1)$ \\
Customised weight centile & $61.6(28.2)$ \\
Sex baby & 22 female, 11 male \\
\hline
\end{tabular}

